\title{
Lactation and appetite-regulating hormones: increased maternal plasma peptide YY concentrations 3-6 months postpartum
}

\author{
Greisa Vila ${ }^{1, *}$, Judith Hopfgartner ${ }^{1}$, Gabriele Grimm ${ }^{2}$, Sabina M. Baumgartner-Parzer ${ }^{1}$, \\ Alexandra Kautzky-Willer ${ }^{1}$, Martin Clodi $^{1}$ and Anton Luger ${ }^{1}$ \\ ${ }^{1}$ Department of Internal Medicine III, Division of Endocrinology and Metabolism, Medical University of Vienna, \\ Vienna 1090, Austria \\ ${ }^{2}$ Department of Medical and Chemical Laboratory Diagnostics, Medical University of Vienna, Vienna 1090, Austria
}

(Submitted 12 October 2014 - Final revision received 27 May 2015 - Accepted 11 June 2015 - First published online 24 August 2015)

\section{Abstract}

Breast-feeding is associated with maternal hormonal and metabolic changes ensuring adequate milk production. In this study, we investigate the impact of breast-feeding on the profile of changes in maternal appetite-regulating hormones 3-6 months postpartum. Study participants were age- and BMI-matched lactating mothers ( $n$ 10), non-lactating mothers $(n 9)$ and women without any history of pregnancy or breast-feeding in the previous 12 months (control group, $n$ 10). During study sessions, young mothers breast-fed or bottle-fed their babies, and maternal blood samples were collected at five time points during $90 \mathrm{~min}$ : before, during and after feeding the babies. Outcome parameters were plasma concentrations of ghrelin, peptide YY (PYY), leptin, adiponectin, prolactin, cortisol, insulin, glucose and lipid values. At baseline, circulating PYY concentrations were significantly increased in lactating mothers $(100.3$ (sE 6.7$) \mathrm{pg} / \mathrm{ml}) v$. non-lactating mothers $(73.6$ (SE 4.9$) \mathrm{pg} / \mathrm{ml}, P=0.008)$ and $v$. the control group $(70.2$ ( $\mathrm{sE} 9) \mathrm{pg} / \mathrm{ml}, P=0.021$ ). We found no differences in ghrelin, leptin and adiponectin values. Baseline prolactin concentrations were over 4 -fold higher in lactating mothers $(P<0 \cdot 001)$. Lactating women had reduced TAG levels and LDL-cholesterol: HDL-cholesterol ratio, but increased waist circumference, when compared with non-lactating women. Breast-feeding sessions further elevated circulating prolactin $(P<0.001)$, but induced no acute effects on appetite-regulating hormones. In summary, one single breast-feeding session did not acutely modulate circulating appetite-regulating hormones, but increased baseline PYY concentrations are associated with prolonged lactation. PYY might play a role in the coordination of energy balance during lactation, increasing fat mobilisation from maternal depots and ensuring adequate milk production for the demands of the growing infant.

\section{Key words: Lactation: Breast-feeding: Appetite: Peptide YY: Ghrelin: Lipids: Cholesterol}

Exclusive breast-feeding during the first 6 months of life increases mother-child bonding and is associated with healthier maternal and offspring outcomes ${ }^{(1-5)}$. Maternal energy metabolism during human lactation is characterised by augmented glucose production and increased mobilisation of fat from maternal depots, ensuring adequate milk production for the increasing demands of the growing infant ${ }^{(6-8)}$. To date, it is not known whether these changes in energy metabolism are related to differences in appetite-regulating hormones.

The neuroendocrine milieu of lactation is governed by increased circulating concentrations of prolactin and oxytocin, as well as inhibition of the gonadotropin-releasing hormone, leading to lactational amenorrhoea ${ }^{(9)}$. These and other hormonal changes during lactation might also affect glucose metabolism, fat metabolism and energy balance. Oxytocin reduces insulin resistance, increases lipolysis and exerts anti-orexigenic effects $^{(10-12)}$. In contrast, prolactin increases the central resistance to leptin, stimulates appetite and promotes high-fat diet-induced obesity ${ }^{(13,14)}$.

Appetite-regulating hormones derived from the gut may also be affected by the complex neuroendocrine changes accompanying lactation, as they are subject to tight regulatory feedbacks within the gut-brain axis and are closely related to alterations of energy metabolism ${ }^{(15,16)}$. Indeed, the gastrointestinal expression and circulating concentrations of the anorexigenic hormone peptide YY (PYY) were increased throughout the lactation period in rodents, whereas ghrelin levels remained unchanged $^{(17)}$. In humans, at 4 weeks postpartum, there were no differences in plasma ghrelin levels and PYY between lactating and non-lactating women ${ }^{(18)}$. Nevertheless, 3 years postpartum, circulating concentrations of both ghrelin and PYY strongly correlated with the duration of breast-feeding ${ }^{(19)}$.

The WHO recommends exclusive breast-feeding during the first 6 months. During this time, the infant gains weight rapidly

Abbreviation: PYY, peptide YY.

* Corresponding author: G. Vila, fax +43 140400 43090, email greisa.vila@meduniwien.ac.at 
and the amount of milk needed for exclusive breast-feeding increases accordingly ${ }^{(20)}$. Therefore, it is expected that hormone differences at 3-6 months postpartum strongly reflect lactationinduced changes and are less influenced by peripartal events. Based on this hypothesis, we studied the effects of breastfeeding on appetite-regulating hormones in a cohort of lactating and non-lactating women 3-6 months postpartum, compared with a control group.

\section{Methods \\ Design and participants}

The protocol was approved by the institutional review board of the Medical University of Vienna and registered with clinicaltrials. gov (NCT00831818). The participants were twenty-nine healthy women, who were recruited after signed informed consent to three age- and BMI-matched groups: lactating group, nonlactating group and control group. All the participants underwent a thorough clinical examination and biochemical tests before being included in the study. The lactating group included ten mothers of 3 to 6-month-old infants (age range 14-23 weeks, mean age 16.2 weeks), who were exclusively breast-fed and had never taken formula milk. The non-lactating group included nine mothers of 3- to 6-month-old infants (age range 14-22 weeks, mean 15.9 weeks), who were not breast-fed during the previous 4 weeks or longer. The control group included ten healthy women with no history of pregnancy or breast-feeding during the previous 12 months. Women who were menstruating (two mothers in the lactating group, all mothers in the non-lactating group and all women in the control group) attended the sessions during the first $3 \mathrm{~d}$ of the follicular phase of the menstrual cycle. Inclusion criteria for lactating and non-lactating groups were as follows: singleton pregnancy, no gestational diabetes, no pregnancy complications, normal vaginal delivery and healthy normally growing infants (reported in the mother-child pass). Exclusion criteria for all three groups were BMI $<18.5 \mathrm{~kg} / \mathrm{m}^{2}$ or BMI $>24.9 \mathrm{~kg} / \mathrm{m}^{2}$, smoking, current endocrine and metabolic diseases, cardiovascular, kidney or liver diseases and malignancy. One lactating mother, five non-lactating mothers and six women from the control group were on hormonal contraception. Four mothers from the breast-feeding group, five mothers from the non-lactating group and four women from the control group were primiparous. Other participants of the lactating and non-lactating group had at least two children. Six participants of the control group were nulliparous.

The recruitment process was performed via flyers. We experienced difficulties in recruiting mothers with normal BMI who did not breast-feed, as $>50 \%$ of not breast-feeding women contacting the study centre were overweight or obese.

During the study day, all the participants came to the clinical research centre between 08.00 and 10.00 hours after fasting for at least $4 \mathrm{~h}$. The timing of the visit was planned to be flexible, as mothers of infants were advised to arrive about $45 \mathrm{~min}$ before the next planned breast-feeding or bottle-feeding episode. Participants from the lactating and non-lactating groups received an intravenous cannula in the left antecubital vein and blood samples were collected at five time points during a time period of at least
90 min: start of the study (time point - $20 \mathrm{~min}$ ), immediately before breast-feeding/bottle-feeding (time point $0 \mathrm{~min}$ ) and 15, 30 and $60 \mathrm{~min}$ after starting breast-feeding/bottle-feeding. Mothers were instructed either to breast-feed their infants (lactating group) or to bottle-feed their infants (non-lactating group). Breast-feeding lasted from 8 to $20 \mathrm{~min}$ (mean $15 \mathrm{~min}$ ).

\section{Assays}

All the study participants provided baseline blood samples for the routine measurement of glucose and lipid values, C-reactive protein, HbA1c and thyroid-stimulating hormone. All these measurements were performed in a routine laboratory (www. kimcl.at). Blood samples for the measurement of ghrelin, PYY, leptin, adiponectin, prolactin, growth hormone (GH), cortisol and insulin were centrifuged for $15 \mathrm{~min}$ at $1200 \mathrm{~g}$, and serum or plasma was frozen at $-20^{\circ} \mathrm{C}$. Blood samples for the measurement of acylated ghrelin were obtained in ice-cold $\left(4^{\circ} \mathrm{C}\right)$ vials containing EDTA and aprotinin (Trasylol, 500000 Kallikrein inhibitor units, $40 \mu \mathrm{l} / \mathrm{ml}$ ). After centrifugation, plasma aliquots of $1 \mathrm{ml}$ were acidified with $110 \mu \mathrm{l} 1 \mathrm{~N}-\mathrm{HCl}$ and immediately frozen at $-20^{\circ} \mathrm{C}$.

All the frozen samples were analysed at the end of the study with kits of the same lot number with all samples obtained from one participant measured within the same kit and an equal proportion of samples from all three groups within each kit. Plasma concentrations of total ghrelin were determined using RIA kits from Peninsula Laboratories (Bachem) with intra-assay and inter-assay $\mathrm{CV}<9.9 \%$. Acylated ghrelin was measured using a sandwich ELISA kit from Linco Research with intra-assay and inter-assay CV both being $<7.5 \%$. Concentrations of PYY, leptin, adiponectin and insulin were determined using RIA kits from Linco Research (Millipore) with intra-assay and inter-assay CV being 9 and $8.2 \%$ for PYY and 5-8\% for leptin and adiponectin, respectively. Prolactin, cortisol and $\mathrm{GH}$ were measured in a routine certified laboratory using electro-chemiluminescence immunoassay, with sensitivity and $\mathrm{CV}$ being $0.05 \mathrm{ng} / \mathrm{ml}$ and $4 \%$ for prolactin, $0.04 \mu \mathrm{g} / \mathrm{dl}$ and $6 \%$ for cortisol and $0.01 \mathrm{ng} / \mathrm{ml}$ and $7.5 \%$ for $\mathrm{GH}$, respectively (www.kimcl.at).

\section{Statistical analysis}

Data are expressed as mean values with their standard errors and were analysed using the statistical software SPSS release 12.0.1 (SPSS Inc.). Baseline characteristics between the three groups were compared by one-way ANOVA followed by multiple $t$ tests with the Bonferroni correction as post hoc statistics, where appropriate. Breast-feeding-induced changes over time were analysed using repeated measurements ANOVA with the interaction between time and treatment (time $\times$ treatment) being the term of interest. $P<0.05$ was considered statistically significant.

\section{Results}

\section{Subject characteristics}

The clinical characteristics of the three groups are given in Table 1. There were no differences in age, weight or BMI 
between the groups. Waist circumference was higher in breastfeeding women (Table 1). Parameters of glucose metabolism were similar in all the groups. Lactating mothers had lower circulating TAG levels and a lower LDL-cholesterol:HDLcholesterol ratio when compared with non-lactating mothers (Table 2).

\section{Baseline circulating hormone levels during lactation}

Full lactation was associated with nearly 4-fold higher circulating prolactin concentrations (Table 3), but no differences in baseline $\mathrm{GH}$, cortisol and insulin levels.

At baseline, there were no differences in circulating concentrations of ghrelin, leptin and adiponectin between the groups (Fig. 1(a, c and d)). However, plasma PYY concentrations were significantly higher in lactating women $(100 \cdot 3(\mathrm{se} 6.7) \mathrm{pg} / \mathrm{ml})$ when compared with non-lactating women $(73.6$ (sE 4.9) pg/ml, $P=0.008$ ) or control women $(70.2$ (sE 9) $\mathrm{pg} / \mathrm{ml}, P=0.021$ ) (Fig. 1(b)).

\section{Profile of hormone changes during breast-feeding sessions}

During the process of breast-feeding, the already basally elevated prolactin concentrations increased further, but there were no changes in the profiles of plasma ghrelin, acylated ghrelin, cortisol and insulin (Fig. 2(a, c-f)). Plasma PYY remained significantly higher throughout the study period (Fig. 2(b)).

\section{Discussion}

The main findings of this study are that full lactation is associated with increased baseline concentrations of maternal PYY, but the act of breast-feeding does not acutely influence the profile of circulating maternal ghrelin and PYY. Furthermore, full lactation is accompanied by increased baseline prolactin concentrations, lower plasma TAG, a better LDL-cholesterol:HDL-cholesterol ratio and increased waist circumference.

To our knowledge, these data on higher PYY concentrations represent the only reported difference in gut-derived hormones during breast-feeding in humans. In reality, the differences in PYY concentrations between lactating and non-lactating

Table 1. Baseline characteristics of study participants (Mean values with their standard errors)

\begin{tabular}{|c|c|c|c|c|c|c|}
\hline & \multicolumn{2}{|c|}{ Lactating } & \multicolumn{2}{|c|}{ Non-lactating } & \multicolumn{2}{|c|}{ Control } \\
\hline & Mean & SE & Mean & SE & Mean & SE \\
\hline Age (years) & 30.5 & $1 \cdot 1$ & $29 \cdot 4$ & 1.9 & $30 \cdot 3$ & 1.5 \\
\hline Weeks postpartum & $16 \cdot 2$ & $1 \cdot 1$ & $15 \cdot 9$ & 1.0 & & \\
\hline Weight (kg) & $62 \cdot 7$ & $2 \cdot 1$ & $59 \cdot 6$ & $2 \cdot 2$ & 61.4 & 1.8 \\
\hline BMI $\left(\mathrm{kg} / \mathrm{m}^{2}\right)$ & $22 \cdot 6$ & 0.6 & $22 \cdot 2$ & 0.6 & $21 \cdot 2$ & 0.4 \\
\hline Waist circumference $(\mathrm{cm})$ & $84 \cdot 8^{*} \dagger$ & $2 \cdot 1$ & $75 \cdot 4^{\star}$ & 1.5 & $72 \cdot 0 \dagger$ & 1.2 \\
\hline Hip (cm) & $100 \cdot 6$ & 2 & $98 \cdot 1$ & 1.7 & $98 \cdot 4$ & 1.3 \\
\hline
\end{tabular}

The lactating group includes women who exclusively breast-feed $(n 10)$, the nonlactating group includes women who had not breast-fed during the previous 4 weeks or longer $(n 9)$ and the control group includes healthy women with no

history of pregnancy or breast-feeding during the previous 12 months ( $n$ 10).

* $P=0.004$ lactating $v$. non-lactating, $+P<0.001$ lactating $v$. control. women might be even greater than that observed in our study, as we compared groups with similar BMI. In general, non-lactating women are more obese than lactating women ${ }^{(21)}$, and obesity is associated with decreased PYY levels ${ }^{(15,16)}$; therefore, the general cohort of non-lactating women is expected to have even lower PYY concentrations.

Lactation is associated with reduced expression of proopiomelanocortin and increased expression of the orexigenic neuropeptide $\mathrm{Y}$ in the hypothalamus, increased prolactin concentrations and decreased oestradiol concentrations ${ }^{(22,23)}$. All these central and peripheral mechanisms impact energy metabolism and might also lead to adaptations in the secretion of gut hormones.

PYY is known for its appetite-inhibitory and lipolytic effects that lead to reduced weight gain in obesity-prone models ${ }^{(24,25)}$. The higher PYY concentrations between the 3rd and the 6th month of lactation are consistent with data from rodent studies, and complement a previous publication reporting no differences in appetite-regulating hormones between lactating and non-lactating women $4-6$ weeks postpartum ${ }^{(17,18)}$. Indeed, the positive effect of lactation on postpartum weight retention is observed after the 3 rd month postpartum, a period which is also less influenced by peripartal events ${ }^{(26)}$. The cross-sectional nature of our study does not allow us to differentiate whether the increase in PYY is a consequence of full lactation or whether reduced PYY is a risk factor for failed lactation. Nevertheless, the fact that PYY concentrations did not differ between lactating and non-lactating mothers at 4 weeks postpartum $^{(18)}$ supports the hypothesis that full prolonged lactation leads to an increase in PYY. This change during the progress of breast-feeding is most likely an adaptation to the concomitant metabolic changes such as the increased energy intake (required for milk production) and mobilisation of maternal depots found to accompany lactation. Indeed, PYY enhances lipolysis and selectively increases carbohydrate consumption, despite reducing appetite ${ }^{(24,25)}$. We hypothesise that elevated PYY levels promote the selective acquisition of nutrients, and enhance utilisation of fat from maternal depots, ensuring adequate milk production for the infant. One of the main reasons for maternal fat mobilisation during breast-feeding is the critical need for long-chain PUFA, arachidonic acid and, especially, DHA, which are limited in the environment, but extremely important for the rapidly growing infant brain ${ }^{(8)}$. Our findings on increased maternal waist circumference and improved plasma lipid profile during breast-feeding are in line with previous studies, which have confirmed an association of breast-feeding with increased waist circumference and a relative decrease in lower-body fat ${ }^{(8,27,28)}$.

We found no differences in ghrelin concentrations during lactation, despite the close relationship between ghrelin and energy metabolism. The main reason for this could be the fact that BMI is the most significant predictor of ghrelin in all the cohorts studied thus far, and we compared three groups with similar BMI in our study ${ }^{(16)}$.

The secretion of ghrelin and PYY follows circadian changes and is strongly influenced by the feeding status. It is important to note that, in this study, we studied breast-feeding-associated differences in appetite-regulating hormones during fasting. In the fasted state, 
Table 2. Parameters of glucose and lipid metabolism (Mean values with their standard errors)

\begin{tabular}{|c|c|c|c|c|c|c|}
\hline & \multicolumn{2}{|c|}{ Lactating } & \multicolumn{2}{|c|}{ Non-lactating } & \multicolumn{2}{|c|}{ Control } \\
\hline & Mean & SE & Mean & SE & Mean & SE \\
\hline Fasting plasma glucose $(\mathrm{mmol} / \mathrm{l})$ & $4 \cdot 33$ & 0.1 & 4.6 & 0.1 & 4.6 & 0.1 \\
\hline HOMA insulin resistance index & 1.61 & 0.13 & 1.99 & 0.18 & 2.04 & 0.4 \\
\hline TAG $(\mathrm{mmol} / \mathrm{l})$ & $0.78^{*}$ & 0.05 & $1.08^{*}$ & 0.08 & 0.98 & 0.11 \\
\hline Total cholesterol ( $\mathrm{mmol} / \mathrm{l})$ & 4.92 & 0.3 & $5 \cdot 6$ & 0.3 & $4 \cdot 81$ & 0.2 \\
\hline LDL-cholesterol (mmol/l) & $2 \cdot 7$ & 0.2 & $3.38 \dagger$ & 0.3 & $2.57 \dagger$ & 0.2 \\
\hline LDL-cholesterol:HDL-cholesterol ratio & $1.5 \ddagger$ & 0.1 & $2 \cdot 1 \neq \S$ & 0.2 & $1.5 \S$ & 0.1 \\
\hline
\end{tabular}

HOMA, homoeostasis model assessment.

The lactating group includes women who exclusively breast-feed $(n 10)$, the non-lactating group includes women who had not breast-fed during the previous 4 weeks or longer ( $n 9$ ) and the control group includes healthy women with no history of pregnancy or breast-feeding during the previous 12 months ( $n 10)$.

${ }^{*} P=0.006$ lactating $v$. non-lactating, $\dagger P=0.025$ non-lactating $v$. control, $\ddagger P=0.036$ lactating $v$. non-lactating, $\S P=0.048$ non-lactating $v$. control.

Table 3. Hormone concentrations of the study subjects

(Mean values with their standard errors)

\begin{tabular}{|c|c|c|c|c|c|c|}
\hline & \multicolumn{2}{|c|}{ Lactating } & \multicolumn{2}{|c|}{ Non-lactating } & \multicolumn{2}{|c|}{ Control } \\
\hline & Mean & SE & Mean & SE & Mean & SE \\
\hline Fasting insulin $(\mu \mathrm{U} / \mathrm{l})$ & $45 \cdot 3$ & $7 \cdot 3$ & $57 \cdot 6$ & 4.6 & $74 \cdot 3$ & $9 \cdot 7$ \\
\hline Prolactin (ng/ml) & $28 \cdot 4^{*} \dagger$ & 3.9 & $6 \cdot 4^{*}$ & 0.9 & $8 \cdot 8^{\star *}$ & $1 \cdot 3$ \\
\hline Cortisol ( $\mu \mathrm{g} / \mathrm{l})$ & 117 & 15 & 126 & 16 & 165 & 21 \\
\hline $\mathrm{GH}(\mathrm{ng} / \mathrm{ml})$ & 6.5 & 1.5 & 5.4 & $2 \cdot 1$ & $5 \cdot 1$ & 0.7 \\
\hline TSH $(\mu \mathrm{U} / \mathrm{ml})$ & 1.96 & 0.4 & 1.83 & 0.4 & 1.99 & 0.3 \\
\hline
\end{tabular}

$\mathrm{GH}$, growth hormone; TSH, thyroid-stimulating hormone.

The lactating group includes women who exclusively breast-feed $(n 10)$, the non-lactating group includes women who had not breast-fed during the previous 4 weeks or longer ( $n 9$ ) and the control group includes healthy women with no history of pregnancy or breast-feeding during the previous 12 months ( $n$ 10).

${ }^{*} P<0.001$ lactating $v$. non-lactating, $† P<0.001$ lactating $v$. control.
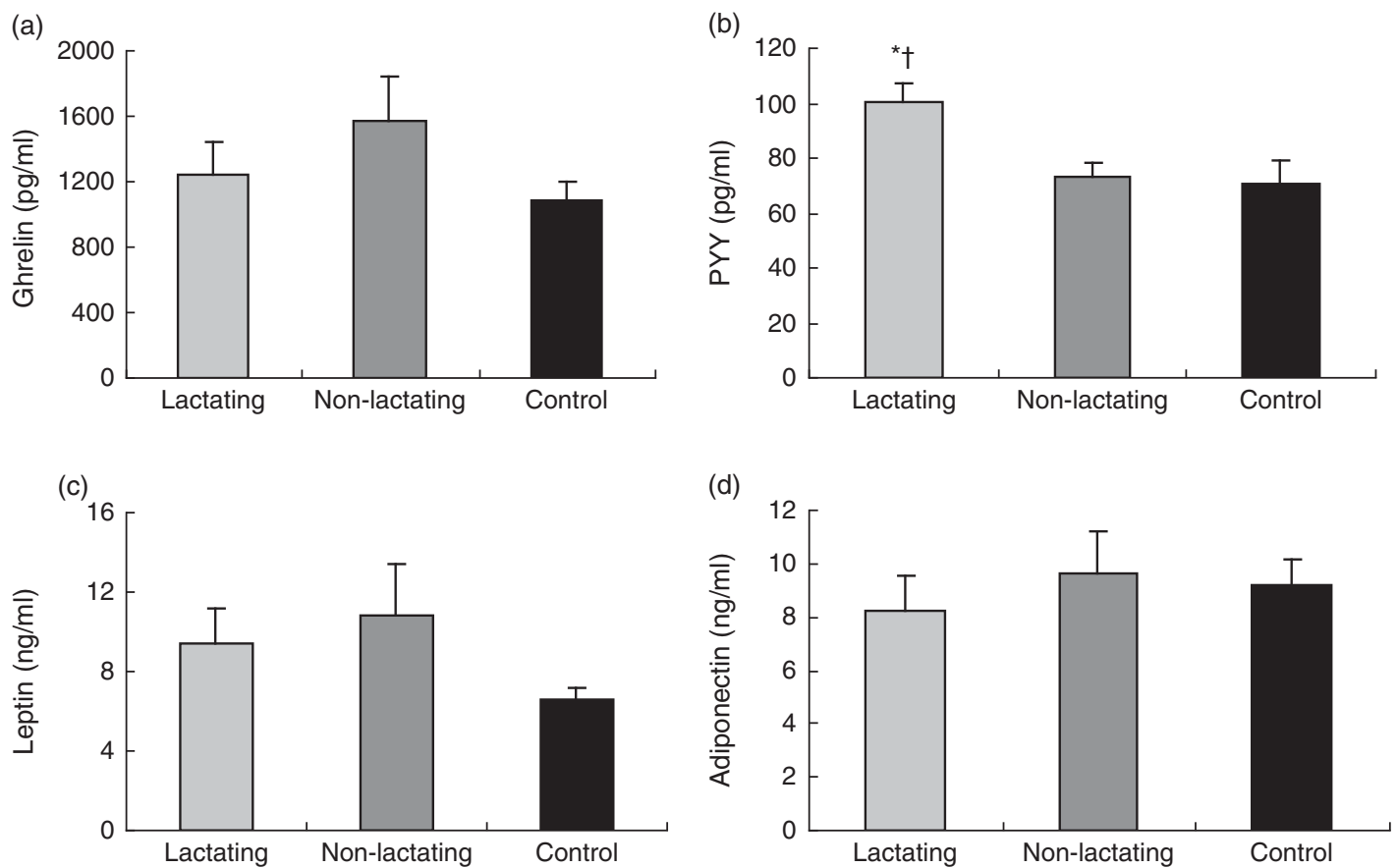

Fig. 1. Baseline concentrations of appetite-regulating hormones. Concentrations of (a) ghrelin, (b) peptide YY (PYY), (c) leptin and (d) adiponectin in the three groups. The lactating group included women who exclusively breast-feed $(n 10)$, the non-lactating group included women who had not breast-fed during the previous 4 weeks or longer $(n 9)$, control group included healthy women with no history of pregnancy or breast-feeding during the previous 12 months $(n 10)$. Data are shown as mean values with their standard errors. ${ }^{*} P=0.008 \mathrm{v}$. non-lactating, $\dagger P=0.021 \mathrm{v}$. control group. 
(a)

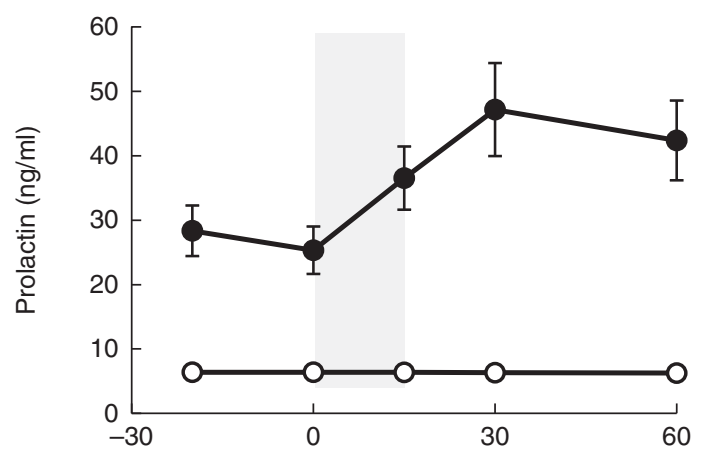

(c)

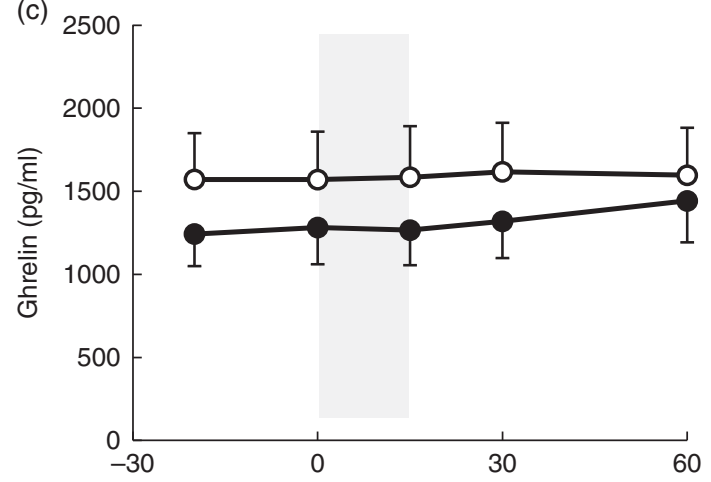

(e)

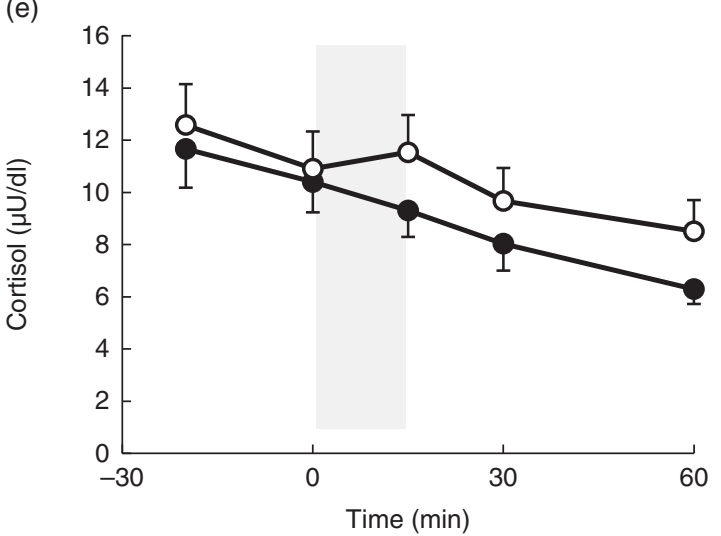

(b)

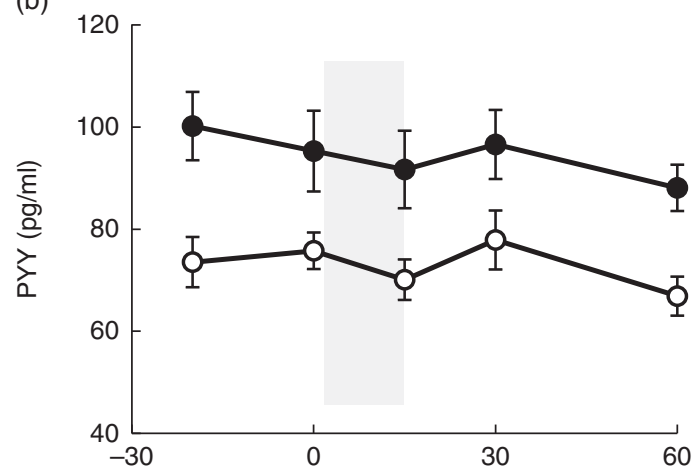

(d)

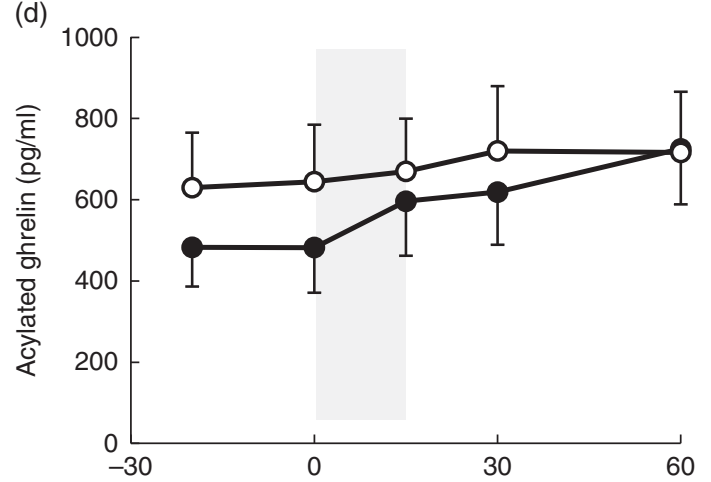

(f)

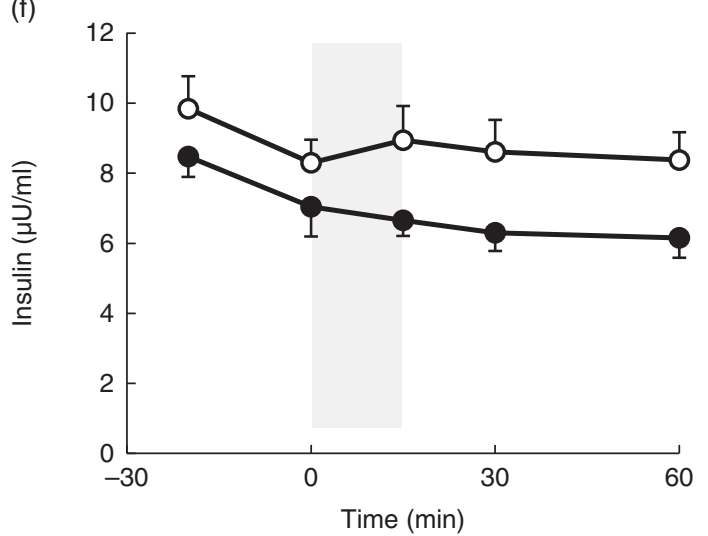

Fig. 2. The profile of hormone changes during breast-feeding. The profile of changes in (a) prolactin, (b) peptide YY (PYY), (c) ghrelin, (d) acylated ghrelin, (e) cortisol and (f) insulin concentrations during breast-feeding $v$. bottle-feeding. $\bullet$, breast-feeding; $O$, bottle-feeding; the shaded area indicates the duration of breast-feeding/ bottle-feeding. Data are shown as mean values with their standard errors.

plasma ghrelin is inversely related to cortisol $^{(29)}$. Despite the well-known reduced hypothalamic-pituitary-adrenal axis activity in response to stressors, as well as changes in the profile of prolactin and oxytocin concentrations during breast-feeding, we did not find any relevant difference in gut-derived appetite-regulating hormones during one single breast-feeding episode $^{(30)}$.

Limitations of this study are the lack of data on maternal education, employment, income, feeding patterns and weight changes over time, as all these parameters could affect the metabolic profile. The strength of this study is the comparison of age- and BMI-matched groups of lactating, non-lactating and control women.

Taken together, in this study, we show that full lactation is associated with elevated baseline PYY concentrations at 3-6 months postpartum, but a single breast-feeding episode does not induce any acute changes in circulating appetiteregulating hormones. Increased plasma PYY concentrations during lactation might not only contribute to increased mobilisation of fat from maternal depots, ensuring adequate milk production in the short term, but also contribute to better maternal health outcomes in the long term. 


\section{Acknowledgements}

The authors thank Astrid Hofer and Liliana-Imi Ionasz for their excellent technical assistance.

The authors declare that they have no conflicting interests.

G. V., A. L. and M. C. designed the research; G. V., J. H., G. G., S. M. B.-P. and A. K.-W. conducted research; G. V., M. C. and A. L. analysed the data; G. V. wrote the manuscript; and G. V. and A. L. had primary responsibility for the final content. All authors read and approved the final manuscript.

\section{References}

1. Beyerlein A \& von Kries R (2011) Breastfeeding and body composition in children: will there ever be conclusive empirical evidence for a protective effect against overweight? Am J Clin Nutr 94, 1772S-1775S.

2. Robinson SM, Marriott LD, Crozier SR, et al. (2009) Variations in infant feeding practice are associated with body composition in childhood: a prospective cohort study. J Clin Endocrinol Metab 94, 2799-2805.

3. Baker JL, Gamborg M, Heitmann BL, et al. (2008) Breastfeeding reduces postpartum weight retention. Am J Clin Nutr 88, 1543-1551.

4. Fagerhaug TN, Forsmo S, Jacobsen GW, et al. (2013) A prospective population-based cohort study of lactation and cardiovascular disease mortality: the HUNT study. BMC Public Health 13, 1070

5. Diniz JM \& Da Costa TH (2004) Independent of body adiposity, breast-feeding has a protective effect on glucose metabolism in young adult women. Br J Nutr $\mathbf{9 2}$, 905-912.

6. Tigas S, Sunehag A \& Haymond MW (2002) Metabolic adaptation to feeding and fasting during lactation in humans. J Clin Endocrinol Metab 87, 302-307.

7. Mohammad MA, Sunehag AL, Chacko SK, et al. (2009) Mechanisms to conserve glucose in lactating women during a 42-h fast. Am J Physiol Endocrinol Metab 297, E879-E888.

8. Lassek WD \& Gaulin SJ (2006) Changes in body fat distribution in relation to parity in American women: a covert form of maternal depletion. Am J Phys Anthropol 131, 295-302.

9. McNeilly AS (2001) Lactational control of reproduction. Reprod Fertil Dev 13, 583-590.

10. Leng G, Onaka T, Caquineau C, et al. (2008) Oxytocin and appetite. Prog Brain Res 170, 137-151.

11. Vila G, Riedl M, Resl M, et al. (2009) Systemic administration of oxytocin reduces basal and lipopolysaccharide-induced ghrelin levels in healthy men. J Endocrinol 203, 175-179.

12. Deblon N, Veyrat-Durebex C, Bourgoin L, et al. (2011) Mechanisms of the anti-obesity effects of oxytocin in diet-induced obese rats. PLOS ONE 6, e25565.

13. Augustine RA \& Grattan DR (2008) Induction of central leptin resistance in hyperphagic pseudopregnant rats by chronic prolactin infusion. Endocrinology 149, 1049-1055.

14. Auffret J, Viengchareun S, Carré N, et al. (2012) Beige differentiation of adipose depots in mice lacking prolactin receptor protects against high-fat-diet-induced obesity. FASEB J 26, 3728-3737.

15. Maier C, Riedl M, Vila G, et al. (2008) Cholinergic regulation of ghrelin and peptide YY release may be impaired in obesity. Diabetes 57, 2332-2340.

16. Murphy KG, Dhillo WS \& Bloom SR (2006) Gut peptides in the regulation of food intake and energy homeostasis. Endocr Rev 27, 719-727.

17. Taylor VJ, Patterson M, Ghatei MA, et al. (2009) Ghrelin and peptide YY (PYY) profiles in gastrointestinal tissues and the circulation of the rat during pregnancy and lactation. Peptides 12, 2213-2220.

18. Larson-Meyer DE, Ravussin E, Heilbronn L, et al. (2010) Ghrelin and peptide YY in postpartum lactating and nonlactating women. Am J Clin Nutr 91, 366-372.

19. Stuebe AM, Mantzoros C, Kleinman K, et al. (2011) Duration of lactation and maternal adipokines at 3 years postpartum. Diabetes 60, 1277-1285.

20. Neville MC, Keller R, Seacat J, et al. (1988) Studies in human lactation: milk volumes in lactating women during the onset of lactation and full lactation. Am J Clin Nutr $\mathbf{4 8}$, $1375-1386$

21. Verret-Chalifour J, Giguère Y, Forest JC, et al. (2015) Breastfeeding initiation: impact of obesity in a large Canadian perinatal cohort study. PLOS ONE 10, e0117512.

22. Chen P, Williams SM, Grove K, et al. (2004) Melanocortin 4 receptor-mediated hyperphagia and activation of neuropeptide $\mathrm{Y}$ expression in the dorsomedial hypothalamus during lactation. J Neurosci 24, 5091-5100.

23. Woodside B (2007) Prolactin and the hyperphagia of lactation. Physiol Behav 91, 375-382.

24. Manning S \& Batterham RL (2014) The role of gut hormone peptide YY in energy and glucose homeostasis: twelve years on. Annu Rev Physiol 76, 585-608.

25. Sloth B, Holst JJ, Flint A, et al. (2007) Effects of PYY1-36 and PYY3-36 on appetite, energy intake, energy expenditure, glucose and fat metabolism in obese and lean subjects. $\mathrm{Am} \mathrm{J}$ Physiol Endocrinol Metab 292, E1062-E1068.

26. Stuebe AM \& Rich-Edwards JW (2009) The reset hypothesis: lactation and maternal metabolism. Am J Perinatol 26, 81-88.

27. Sohlstrom A \& Forsum E. (1995) Changes in adipose tissue volume and distribution during reproduction in Swedish women as assessed by magnetic resonance imaging. Am J Clin Nutr 61, 287-295.

28. Brewer MM, Bates MR \& Vannoy LP. (1989)) Postpartum changes in maternal weight and body fat depots in lactating vs nonlactating women. Am J Clin Nutr 49, 259-265.

29. Espelund U, Hansen TK, Højlund K, et al. (2005) Fasting unmasks a strong inverse association between ghrelin and cortisol in serum: studies in obese and normal-weight subjects. J Clin Endocrinol Metab 90, 741-746.

30. Heinrichs M, Meinlschmidt G, Neumann I, et al. (2001) Effects of suckling on hypothalamic-pituitary-adrenal axis responses to psychosocial stress in postpartum lactating women. J Clin Endocrinol Metab 86, 4798-4804. 